\title{
Immunohistochemical analysis of MDM2 and CDK4 distinguishes low-grade osteosarcoma from benign mimics
}

\author{
Akihiko Yoshida ${ }^{1,2}$, Tetsuo Ushiku ${ }^{2}$, Toru Motoi ${ }^{3}$, Tatsuhiro Shibata ${ }^{4}$, Yasuo Beppu ${ }^{5}$, \\ Masashi Fukayama ${ }^{2}$ and Hitoshi Tsuda ${ }^{1}$ \\ ${ }^{1}$ Clinical Laboratory Division, National Cancer Center Hospital, Tokyo, Japan; ${ }^{2}$ Department of Pathology, \\ University of Tokyo, Tokyo, Japan; ${ }^{3}$ Department of Pathology, Teikyo University, Tokyo, Japan; ${ }^{4}$ Cancer \\ Genomics Project, Center for Medical Genomics, National Cancer Center Research Institute, Tokyo, Japan \\ and ${ }^{5}$ Department of Orthopedics, National Cancer Center Hospital, Tokyo, Japan
}

\begin{abstract}
Parosteal osteosarcoma and low-grade central osteosarcoma are two types of low-grade osteosarcoma that show similar clinical behaviors, histological features, and genetic background (ie, amplified sequences of 12q13-15, including MDM2 and CDK4). Low-grade osteosarcoma is often confused with benign lesions, and ancillary techniques to enhance diagnostic accuracy have been awaited. This study explores the use of MDM2 and CDK4 immunohistochemistry for the histological diagnosis of low-grade osteosarcoma. We studied 23 cases of low-grade osteosarcoma from 21 patients (parosteal osteosarcoma $(n=14)$, low-grade central osteosarcoma $(n=9)$ ) and 40 cases of benign histological mimics (myositis ossificans $(n=11)$, fibrous dysplasia $(n=14)$, osteochondroma $(n=6)$, desmoplastic fibroma $(n=1)$, florid reactive periostitis $(n=4)$, Nora's lesion $(n=3)$, and turret exostosis $(n=1))$. Low-grade osteosarcoma labeled for MDM2 in 16 cases $(70 \%)$ and for CDK4 in 20 cases (87\%). All low-grade osteosarcomas expressed one or both markers (100\%), with 13 cases $(57 \%)$ expressing both. Staining pattern was diffuse in most cases, and the majority expressed moderate or strong intensity for either antibody. MDM2/CDK4 immunostaining was shown irrespective of low-grade osteosarcoma histological subtype. In contrast, only 1 Nora's lesion out of the $\mathbf{4 0}$ miscellaneous benign processes showed immunoreactivity for MDM2 or CDK4. The combination of these two markers thus shows $100 \%$ sensitivity and $97.5 \%$ specificity for the diagnosis of low-grade osteosarcoma. MDM2 and CDK4 immunostains therefore reliably distinguish low-grade osteosarcoma from benign histological mimics, and their combination may serve as a useful adjunct in this difficult differential diagnosis.
\end{abstract}

Modern Pathology (2010) 23, 1279-1288; doi:10.1038/modpathol.2010.124; published online 2 July 2010

Keywords: CDK4; immunohistochemistry; low-grade central osteosarcoma; MDM2; parosteal osteosarcoma

Parosteal osteosarcoma and low-grade central osteosarcoma are two subtypes of low-grade osteosarcoma, comprising less than $5 \%$ of all osteosarcomas. ${ }^{1}$ Parosteal osteosarcoma typically arises on the surface of the long tubular bone with a characteristic lobulated plastered-on appearance, culminating in envelopment of the host bone. ${ }^{2-5}$ On the other hand, low-grade central osteosarcoma develops

Correspondence: Dr A Yoshida, MD, Clinical Laboratory Division, National Cancer Center Hospital, 5-1-1 Tsukiji, Chuo-ku, Tokyo, Japan.

E-mail: ayoshida-tky@umin.ac.jp

Received 14 April 2010; revised 28 May 2010; accepted 31 May 2010; published online 2 July 2010 within the intramedullary cavity often of the long tubular bone. ${ }^{2-5}$ Despite the difference in location relative to the parent bone, it is well established that both tumors have many common features. ${ }^{1}$ They both affect young adults, with the peak incidence in the third decade of life, ${ }^{2-4}$ a decade older than conventional osteosarcoma that typically occurs in teenagers. They manifest as a mass (parosteal) or as pain/swelling (central) of longer duration than conventional osteosarcoma. ${ }^{2-5}$ The similarity extends to the histological level in that both consist of coarse bony trabeculae admixed with hypocellular mildly atypical spindle cell proliferations in fibrotic stroma. ${ }^{2-4}$ Furthermore, these low-grade osteosarcoma variants behave in a similar manner. ${ }^{1-5}$ They may 
recur after the resection, and almost invariably do so when incompletely excised. Although they have virtually no intrinsic capacity to metastasize ${ }^{5-7}$ they can progress to higher-grade sarcomas as 'dedifferentiation' process to gain a metastatic risk as high as that of conventional high-grade osteosarcoma. ${ }^{4}$ Some $15-40 \%$ of low-grade osteosarcoma cases are reported to contain foci of dedifferentiation. ${ }^{1,6,8}$ A recent large comparison study showed that parosteal and low-grade central osteosarcoma have similar rates of local recurrence, metastasis, dedifferentiation, and disease-specific survival. ${ }^{1}$

Besides the above-described clinicopathological resemblance, recently accumulated cytogenetic and molecular genetic data on parosteal and low-grade central osteosarcomas disclosed their shared genetic background. Parosteal osteosarcoma was repeatedly shown to harbor one or more supernumerary ring chromosomes, often as the sole abnormality. ${ }^{9-12}$ This low number of chromosomal imbalances is in contrast with the complex numerical and structural aberrations observed in conventional osteosarcoma. ${ }^{3}$ The ring chromosomes of parosteal osteosarcoma were found, by comparative genomic hybridization, to carry amplified $12 q 13-15$ genetic material. ${ }^{12,13}$ Low-grade central osteosarcoma was also shown to have amplified chromosome 12 with $12 q 13-15$ as the minimal common region. ${ }^{14}$ Subsequent analyses by Southern blotting, quantitative polymerase chain reaction, and/or fluorescence in situ hybridization identified amplified genes such as MDM2, SAS, and CDK4 in this chromosome segment. ${ }^{11,15-20}$ These results suggest that the similarity between parosteal and low-grade central osteosarcomas stems from the genetic level, further strengthening the relationship between the two tumor subtypes. ${ }^{1,14}$

Diagnosis of low-grade osteosarcoma is challenging. Because of its protracted clinical course and deceptively bland histological appearance, lowgrade osteosarcoma is often confused with benign lesions, most often fibrous dysplasia or myositis ossificans, ${ }^{2,5}$ and the confusion can be bidirectional. Low-grade osteosarcoma diagnosis therefore requires scrupulous analysis of histology, radiology, and clinical information, and the particular focus should be given to the history of trauma, lesional growth rate, evidence of invasion, nuclear atypia, architectural pattern such as 'zonation', shape of bone trabeculae, and osteoblastic rimming. ${ }^{2-5}$ The differential diagnosis between low-grade osteosarcoma and benign mimics is further complicated by the wide gross/histological variability of each entity. Low-grade osteosarcoma, for example, may show a total lack of nuclear atypia, ${ }^{5}$ 'fibrous dysplasia-like' delicate pattern of bone deposition, ${ }^{7,21}$ extensive fibrous zone resembling desmoplastic fibroma, ${ }^{7,21}$ prominent cartilage cap imitating osteochondroma, ${ }^{22}$ and focal osteoblastic rimming. The variability on the part of benign mimics includes a rare exophytic localization of fibrous dysplasia, ${ }^{23}$ long tubular bone involvement by bizarre parosteal osteochondromatous proliferation and florid reactive periostitis, ${ }^{24}$ nuclear atypia in bizarre parosteal osteochondromatous proliferation and florid reactive periostitis, ${ }^{25}$ incomplete or absent 'zonation' in myositis ossificans, ${ }^{25}$ and degenerative nuclear atypia ('pseudoanaplasia') in fibrous dysplasia. ${ }^{5,26}$ Reflecting the inherent diagnostic difficulty of lowgrade osteosarcoma, the literature is replete with a number of examples initially managed improperly based on erroneous labeling. Because low-grade osteosarcoma requires a distinct treatment from benign lesions, ancillary techniques to enhance diagnostic accuracy have been keenly awaited.

Ancillary studies to separate low-grade osteosarcoma and benign mimics have been explored only twice in the literature, and the subjects were limited to the intramedullary variety in both instances. Okada et $a l^{27}$ used silver-staining nucleolar organizer regions and MIB-1 immunostain to separate lowgrade central osteosarcoma and fibrous dysplasia, and found that sarcoma showed a significantly higher labeling index. This approach may be helpful in selected cases, but we have experienced difficulties in the application of this method in several instances; the MIB-1 indices of low-grade central osteosarcomas were low, in the range of $0-1 \%$, possibly due to decalcification, and were noncontributory to the original diagnoses. In addition, this method may not be readily adoptable in the differential diagnosis of parosteal osteosarcoma and benign processes, because myositis ossificans and other benign surface lesions unfortunately tend to show some proliferative activity, and their increased labeling indices may overlap with those of sarcoma. Pollandt and co-workers ${ }^{28}$ on the other hand, took a molecular approach by performing $G s \alpha$ gene mutation analysis to separate low-grade central osteosarcoma and fibrous dysplasia. They found that all nine fibrous dysplasias harbored this mutation, whereas such abnormality was not identified in four of five examples of fibrous dysplasialike low-grade central osteosarcoma. This method is also inapplicable to the surface lesions, and it is too costly and labor-intensive to be used in routine diagnostic practice.

A few previous studies have shown that high rate of gene amplification of MDM2 and CDK4 in lowgrade osteosarcoma is translated to the immunohistochemical overexpression of their protein products. Gamberi et $a l^{15}$ showed that $92 \%(23 / 25)$ parosteal osteosarcomas were immunohistochemically positive for CDK4 and 40\% (10/25) for MDM2. One parosteal osteosarcoma arising from the jaw was shown to express strong MDM2 and CDK4 immunopositivity. ${ }^{29}$ Likewise, Park et al ${ }^{19}$ showed MDM2 immunoreactivity in 33\% low-grade central osteosarcoma samples $(7 / 21)$. In the study by Ragazzini et $a l^{30} 35 \%(7 / 20)$ and $65 \%(13 / 20)$ of low-grade central osteosarcoma were labeled for MDM2 and CDK4, respectively. However, these immunostains have not been tested in the benign histological 
mimics of low-grade osteosarcoma, and thus, have never been explored as a potential diagnostic adjunct of this sarcoma. In this study, we investigate the immunohistochemical expression of MDM2 and CDK4 both in low-grade osteosarcomas and in benign histological mimics, to determine if these markers are of any help in this challenging differential diagnosis.

\section{Materials and methods}

\section{Case Selection}

A retrospective search for low-grade osteosarcoma was performed on both in-house and departmental consultation files of the National Cancer Center Hospital, University of Tokyo Hospital, and Teikyo University Hospital. We also sought for benign lesions that may histologically simulate low-grade osteosarcoma. The entities that potentially mimic low-grade osteosarcoma, based on the literature and our own experience, include fibrous dysplasia, myositis ossificans (heterotopic ossification), florid reactive periostitis, bizarre parosteal osteochondromatous proliferation (also known as Nora's lesion), turret exostosis, desmoplastic fibroma, and osteochondroma. The diagnoses were verified with well-described criteria. $^{2-4}$ Specifically, parosteal osteosarcoma and low-grade central osteosarcoma are fibroblastic osteosarcoma, composed of spindle cells in dense collagenous stroma associated with mature bone. Predominantly osteoblastic or chondroblastic osteosarcomas were not included in the study regardless of nuclear grade or quantity of matrix. The authors also took care to use only cases with unequivocal diagnoses. The latter was achieved by a combination of adequate number of glass slides for review, detailed gross description and/or photographs, clinical data including followups, and radiological information. Three cases of diagnostic uncertainty were excluded, which received conflicting opinions from several bone pathologists. Consequently, 23 cases of low-grade osteosarcomas from 21 patients (parosteal osteosarcoma $(n=14)$, low-grade central osteosarcoma $(n=9))$ met our inclusion criteria, and 40 cases of benign histological simulants (myositis ossificans $(n=11)$, fibrous dysplasia $(n=14)$, osteochondroma $(n=6)$, desmoplastic fibroma $(n=1)$, florid reactive periostitis $(n=4)$, bizarre parosteal osteochondromatous proliferation $(n=3)$, turret exostosis $(n=1))$ were collected for comparison. This study was approved by the institutional review board of the participating institutions.

\section{Immunohistochemistry}

\section{Preparation}

A block containing the largest representative section of each lesion was selected. For sarcomas with dedifferentiation, we studied only low-grade areas. Sections ( $4 \mu \mathrm{m}$ thick) from the paraffin block were routinely deparaffinized. The sections were exposed to $3 \%$ hydrogen peroxide for $15 \mathrm{~min}$ to block endogenous peroxidase activity and then washed in deionized water for 2-3 min. Preparations were pretreated with TRS (Dako, Glostrup, Denmark) for MDM2 and with citrate buffer $(0.01 \mathrm{M}$ citric acid, $\mathrm{pH}$ 6.0) for $\mathrm{CDK} 4$, and autoclaved for antigen retrieval. The primary antibodies used were MDM2 (IF2, dilution 1:100; Zymed Laboratories, San Francisco, CA, USA) and CDK4 (DCS-31, dilution 1:200; Biosource International, Camarillo, CA, USA). The slides were allowed to react by incubating for $1 \mathrm{~h}$ at room temperature with the above primary antibodies and subsequent labeling with the Envision + /HRP system (Dako). Diaminobenzidine was used as the chromogen, and hematoxylin as the counterstain. Appropriate positive and negative controls were run.

\section{Interpretation}

The evaluation was performed on lesional spindle cells. Only nuclear stains were considered positive for MDM2 and CDK4. CDK4 usually stained both nuclei and cytoplasms. Because conventional osteochondroma lacked spindle cell population, the staining of lesional chondrocytes in cartilaginous caps was assessed instead. The spindle cells in fibrous dysplasia uncommonly labeled with MDM2 in their cytoplasm as paranuclear dot pattern; however, this signal was disregarded because it was not nuclear. In addition, the nuclei of osteoclasts, osteoclast-like giant cells, histiocytes, and degenerated skeletal muscle cells sometimes showed weak MDM2 nuclear reactivity; however, the round regular nuclear shape of such cells was readily distinguishable from that of the lesional spindle cell component. The intensity of staining was semiquantitatively graded: 0 , negative; $1+$, weak positive; $2+$, moderately positive; $3+$, strongly positive. The extent of staining was classified as $0-<1 \%$ (negative), $1-10 \%$ (focal), and $>10-$ $100 \%$ (diffuse).

\section{Influence of Decalcification on MDM2/CDK4 Immunohistochemistry}

To determine the influence of decalcification on MDM2 and CDK4 immunoreactivity, we prepared serial sections from a dedifferentiated liposarcoma (a known positive control of MDM2 and CDK4), decalcified them by Plank-Rychlo's solution (a mixture of $70 \mathrm{~g}$ aluminum chloride, $50 \mathrm{ml}$ formic acid, $85 \mathrm{ml} 37 \%$ hydrochloric acid, and $100 \mathrm{ml}$ distilled water) at room temperature for a series of durations (1 day, 1 week, and 2 weeks), and assessed the MDM2/CDK4 immunoreactivity of each section. The results were expressed with the intensity (weak to strong) and the percentage of the stained tumor cells. The experiment was repeated using a different case of dedifferentiated liposarcoma. 


\section{Results}

\section{Clinicopathological Findings of Low-Grade Osteosarcomas}

Pertinent clinicopathological findings of the lowgrade osteosarcomas are summarized in Table 1 . The patients were mostly in their third to fourth decades (median, 29 years old; range, 13-70 years) with a 9:14 male (M) to female (F) ratio. The affected sites were extremities in 18 cases, axial bones in 2 cases, and craniofacial bones in 3 cases. The lesions had been present for relatively long periods up to more than 20 years. Nine tumors were low-grade central osteosarcomas and fourteen were parosteal osteosarcomas; their demographics were similar (central: median, 27 years old; $\mathrm{M}: \mathrm{F}=3: 6$; parosteal: median, 29.5 years old; $M: F=6: 8$ ). The median size of the tumors was $8.3 \mathrm{~cm}$ (range, $2.5-20 \mathrm{~cm}$ ). The radiological findings of parosteal osteosarcoma were rather uniform, presenting as a dense sclerotic mass plastered on the cortex (Figure 1a), whereas those of low-grade central osteosarcoma were less uniform but were often manifested as relatively well-circumscribed lytic and sclerotic lesions in the medulla, with focal trabeculated appearance and frequent bone expansion (Figure 1b).

All low-grade osteosarcomas showed similar histological appearances. They were composed of mildly to moderately cellular fibroblast-like spindle cell proliferation in fibrosclerotic stroma admixed
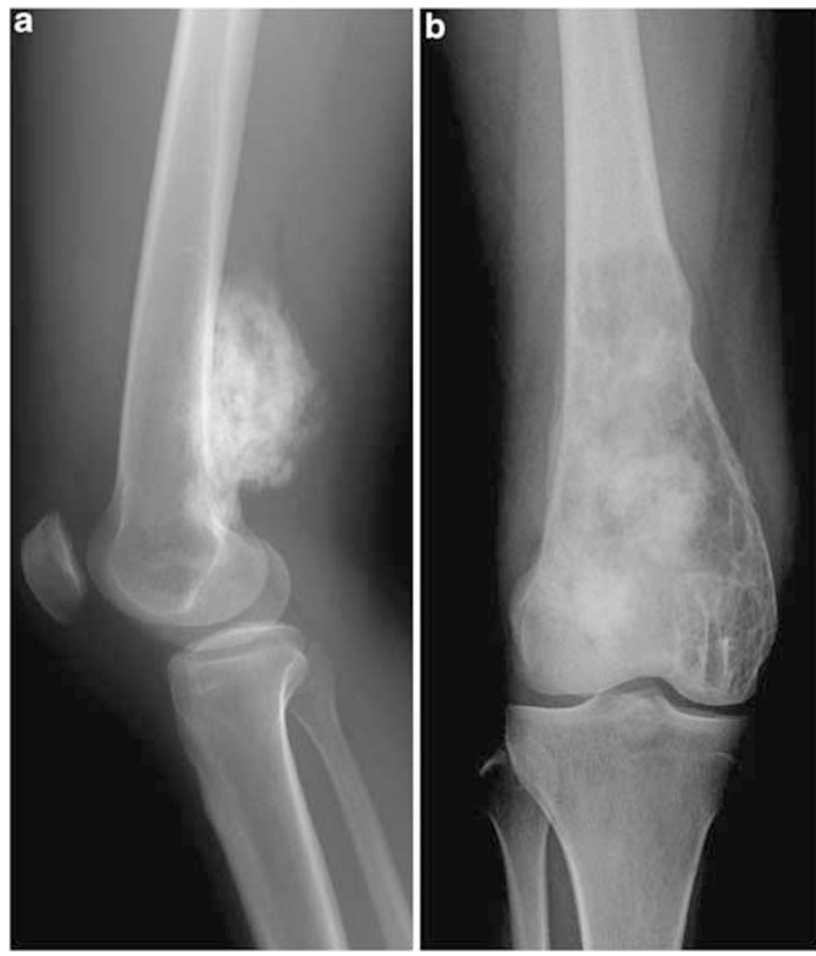

Figure 1 (a) Radiological appearance of a typical parosteal osteosarcoma (case 2) showing dense sclerotic mass plastered on the surface of the posterior femoral cortex. (b) Radiological appearance of a typical low-grade central osteosarcoma (case 16) showing relatively well-circumscribed mixed lytic and sclerotic lesion with trabeculation in the expanded femoral metaphysis.

Table 1 Clinicopathological features of low-grade osteosarcoma

\begin{tabular}{|c|c|c|c|c|c|c|c|c|c|}
\hline No. & Age/Sex & Site & Size $(\mathrm{cm})$ & Type & $\begin{array}{l}\text { Suggested } \\
\text { benign Dx }\end{array}$ & $\begin{array}{c}\text { Initial } \\
\text { management }\end{array}$ & $\begin{array}{c}\text { Final } \\
\text { treatment }\end{array}$ & $D D$ & $\begin{array}{l}\text { Follow-up } \\
\text { (years) }\end{array}$ \\
\hline 1 & $32 / \mathrm{F}$ & Femur & 5.5 & $\mathrm{P}$ & - & - & WE & SD & NED (2) \\
\hline 2 & $28 / \mathrm{F}$ & Femur & 8.0 & $\mathrm{P}$ & - & - & WE & - & NED (3) \\
\hline 3 & $23 / F$ & Femur & 6.0 & $\mathrm{P}$ & Exophytic FD & Observation & WE & - & NED (7) \\
\hline 4 & $66 / \mathrm{M}$ & Ulna & 13.5 & $\mathrm{P}$ & - & - & WE & - & NED (12) \\
\hline $5 a$ & $21 / \mathrm{M}$ & Femur & 8 & $\mathrm{P}$ & OC & ME & - & - & $\operatorname{Rec}(3)$ \\
\hline $5 b$ & $24 / \mathrm{M}$ & Femur & 3 & $\mathrm{P}$ & - & - & WE & - & NED (4) \\
\hline 6 & $45 / F$ & Femur & 4 & $\mathrm{P}$ & 'Benign' & $\mathrm{ME} \times 3$ & WE & - & NED (26) \\
\hline 7 & $39 / F$ & Radius & 20 & $\mathrm{P}$ & $\mathrm{DF}$ & $\mathrm{ME}$ & WE & - & NA \\
\hline 8 & $24 / \mathrm{M}$ & Femur & 7.5 & $\mathrm{P}$ & - & - & WE & $\mathrm{MD}$ & $\operatorname{Rec} \times 3$, DOD $(\mathrm{NA})$ \\
\hline 9 & $70 / \mathrm{M}$ & Ilium & 10 & $\mathrm{P}$ & MO & Observation & Supportive & MD & DOD $(0.6)$ \\
\hline 10 & $23 / F$ & Femur & 11 & $\mathrm{P}$ & - & - & WE & - & NA \\
\hline $11 \mathrm{a}$ & $29 / \mathrm{F}$ & Tibia & NA & $\mathrm{P}$ & - & ME & - & - & $\operatorname{Rec}(1)$ \\
\hline $11 b$ & $30 / F$ & Tibia & NA & $\mathrm{P}$ & - & - & WE & - & NED (18) \\
\hline 12 & $34 / \mathrm{M}$ & Humerus & 8.8 & $\mathrm{P}$ & - & - & WE & - & NA \\
\hline 13 & $51 / \mathrm{F}$ & Maxilla & 2.5 & $\mathrm{C}$ & 'Benign’' & Enucleation & WE & - & NED (2) \\
\hline 14 & $24 / \mathrm{F}$ & Femur & 8 & $\mathrm{C}$ & FD & Curettage & $\mathrm{CT}+\mathrm{WE}$ & - & NED (10) \\
\hline 15 & $32 / \mathrm{F}$ & Humerus & 10 & $\mathrm{C}$ & - & - & $\mathrm{CT}+\mathrm{WE}$ & SD & NED (1) \\
\hline 16 & $27 / F$ & Femur & 11 & $\mathrm{C}$ & - & - & $\mathrm{CT}+\mathrm{WE}$ & SD & Met (1), AWD (2) \\
\hline 17 & $13 / \mathrm{F}$ & Temporal & 4 & $\mathrm{C}$ & FD & Observation & NA & - & NA \\
\hline 18 & $68 / \mathrm{F}$ & Ischium & 9.4 & $\mathrm{C}$ & - & - & WE & MD & $\operatorname{Rec} \times 6, \operatorname{Met}(3)$, AWD (5) \\
\hline 19 & $35 / \mathrm{M}$ & Mandible & NA & $\mathrm{C}$ & - & - & $\mathrm{ME}+\mathrm{CT}+\mathrm{RT}$ & MD & DOD (9) \\
\hline 20 & $18 / \mathrm{M}$ & Femur & 8.5 & $\mathrm{C}$ & FD & Curettage & NA & - & NA \\
\hline 21 & $16 / \mathrm{M}$ & Tibia & 8.5 & $\mathrm{C}$ & - & - & $\mathrm{WE}+\mathrm{CT}$ & SD & NED (3) \\
\hline
\end{tabular}

M, male; F, female; C, low-grade central; P, parosteal; Dx, diagnosis; FD, fibrous dysplasia; DF, desmoplastic fibroma; MO, myositis ossificans; OC, osteochondroma; WE, wide excision; ME, marginal excision; CT, chemotherapy; RT, radiotherapy; DD, dedifferentiation; SD, synchronous dedifferentiation; MD, metachronous dedifferentiation; NED, no evidence of disease; DOD, dead of disease; AWD, alive with disease; Met, metastasis; Rec, recurrence; NA, data not available.

$5 \mathrm{~b}$ and $11 \mathrm{~b}$ are recurrences of $5 \mathrm{a}$ and $11 \mathrm{a}$, respectively. 
with thick bone. The osseous components consisted of long interanastomosing structures with occasional somewhat parallel distribution (Figure 2a). They were woven in type, but were sometimes remodeled into a lamellar variety. Tumor spindle cells showed mild atypia (Figure 2b), including hyperchromasia and nuclear membrane irregularity (18 cases were grade 1 , whereas 5 cases were grade 2 in Dahlin's system ${ }^{31}$ ). Mitotic figures were rare or absent. Tumor spindle cells typically ran parallel to the bone, and they were gradually embedded into the trabeculae at the edges of the neoplastic bone. Cartilaginous components were seen in seven cases, of which two (cases 5a and 5b, a lesion and its recurrence) closely resembled osteochondromas. Osteoblastic rimming was focally present in six cases. In five cases (cases 3, 6, 7, 16, and 19), bone formation was focally absent and the tumor was composed of purely fibrotic tissue with coarse fragments of collagen and thin-walled gaping vessels, resembling desmoplastic fibroma. In three
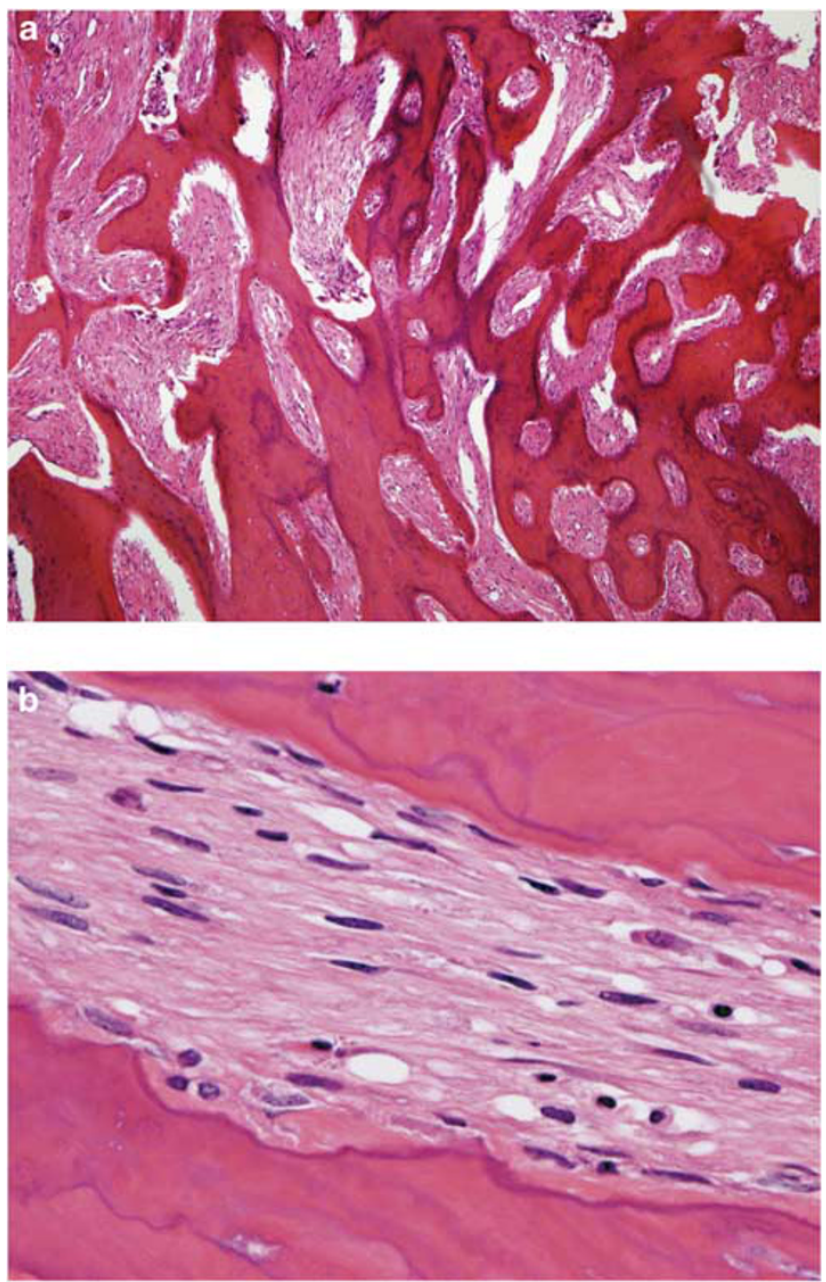

Figure 2 The typical histological appearance of low-grade osteosarcoma, consisting of fascicles of long spindle cells with bland nuclei admixed with mature bone trabeculae in a somewhat parallel array (a: case 3 , b: case 8). cases (cases 3, 14, and 20), tumor bones were thinner and irregular, often tethered by spike-like 'Sharpey fibers', and the findings were reminiscent of fibrous dysplasia (Figure 4, left). Fat was present in nine cases associated with the osseous component. Hematopoietic elements were not seen. Osteoclast-like giant cells were present in three cases. Infiltrative spindle cells either into the adjacent marrow space or skeletal muscle were identified in all the cases except for two biopsy samples (cases 9 and 17). Dedifferentiation to higher grade occurred in eight cases; such foci were present in the original excisional specimens in four cases (synchronous dedifferentiation) and in subsequent excisions (or autopsy materials) in four cases (metachronous dedifferentiation). Nine tumors (39\%) were initially misinterpreted as benign processes on histological/ cytological examinations, and they were first treated conservatively. The final treatment procedures were known in 19 patients: supportive treatment in 1 case, marginal excision in 1 case, and wide excision in 17 cases. Chemotherapy and radiotherapy were used in five cases, four of which showed evidence of dedifferentiation. Follow-up information was available in 16 patients. Nine patients experienced no recurrence after the initial excisions, whereas the remaining seven patients had local progression, recurrences, or metastases. All five patients who either died of disease or suffered from distant metastases had dedifferentiation.

\section{MDM2 and CDK4 Expression Status}

Immunohistochemical results of low-grade osteosarcomas along with pathological findings are seen in Table 2. Low-grade osteosarcoma labeled for MDM2 in 16 cases $(70 \%)$ and for CDK4 in 20 cases $(87 \%)$. All the low-grade osteosarcomas expressed one or both the markers $(100 \%)$, with 13 cases expressing both (57\%) (Figure 3 left and Figure 4 left). A diffuse staining pattern was observed in most tumors (21/ $23,91 \%)$, and the majority $(17 / 23,74 \%)$ of the cases expressed moderate or strong intensity for either antibody. The staining tended to be stronger in tumor cells with grade 2 nuclear atypia than those with grade 1 atypia even in the same tumor. The tumor cells associated with bone matrix (ie, osteoblasts and osteocytes) were also immunoreactive, but the staining was more focal and weaker than fibroblastic tumor cells in the stroma. In one osteochondroma-like parosteal osteosarcoma and its recurrence, not only spindle cells but tumor chondrocytes also labeled for these markers (Figure 5). Tumors with wide areas of fibrous growth without bone formation (desmoplastic fibromalike pattern) were also reactive to either marker (Figure 6). Parosteal osteosarcomas were positive for MDM2 in 57\% (8/14) of the cases, and for CDK4 in $100 \%(14 / 14)$ of the cases. Fifty-seven percent (8/14) of parosteal osteosarcomas were labeled for both 
Table 2 MDM2/CDK4 immunohistochemistry of low-grade osteosarcomas

\begin{tabular}{|c|c|c|c|c|c|c|}
\hline$\#$ & $\begin{array}{c}\text { Age/ } \\
\text { Sex }\end{array}$ & Type & $\begin{array}{c}\text { MDM2 } \\
\text { IHC }\end{array}$ & $\begin{array}{c}\text { CDK4 } \\
\text { IHC }\end{array}$ & $\begin{array}{c}\text { Nuclear } \\
\text { Grade }\end{array}$ & DD \\
\hline 1 & $32 / \mathrm{F}$ & $\mathrm{P}$ & $2 \mathrm{D}$ & $3 \mathrm{D}$ & 2 & + \\
\hline 2 & $28 / \mathrm{F}$ & $\mathrm{P}$ & $1 \mathrm{~F}$ & $2 \mathrm{D}$ & 1 & - \\
\hline 3 & $23 / \mathrm{F}$ & $\mathrm{P}$ & 0 & $2 \mathrm{D}$ & 1 & - \\
\hline 4 & $66 / \mathrm{M}$ & $\mathrm{P}$ & 0 & $2 \mathrm{D}$ & 2 & - \\
\hline $5 \mathrm{a}$ & $21 / \mathrm{M}$ & $\mathrm{P}$ & $1 \mathrm{D}$ & $2 \mathrm{D}$ & 1 & - \\
\hline $5 \mathrm{~b}$ & $24 / \mathrm{M}$ & $\mathrm{P}$ & 0 & $1 \mathrm{D}$ & 1 & - \\
\hline 6 & $45 / \mathrm{F}$ & $\mathrm{P}$ & $1 \mathrm{D}$ & $3 \mathrm{D}$ & 1 & - \\
\hline 7 & $39 / \mathrm{F}$ & $\mathrm{P}$ & 0 & $1 \mathrm{D}$ & 1 & - \\
\hline 8 & $24 / \mathrm{M}$ & $\mathrm{P}$ & $2 \mathrm{D}$ & $2 \mathrm{D}$ & 1 & + \\
\hline 9 & $70 / \mathrm{M}$ & $\mathrm{P}$ & $3 \mathrm{D}$ & $3 \mathrm{D}$ & 1 & + \\
\hline 10 & $23 / \mathrm{F}$ & $\mathrm{P}$ & $2 \mathrm{D}$ & $1 \mathrm{D}$ & 2 & - \\
\hline $11 \mathrm{a}$ & $29 / \mathrm{F}$ & $\mathrm{P}$ & $2 \mathrm{~F}$ & $1 \mathrm{D}$ & 1 & - \\
\hline $11 \mathrm{~b}$ & $30 / \mathrm{F}$ & $\mathrm{P}$ & 0 & $1 \mathrm{D}$ & 1 & - \\
\hline 12 & $34 / \mathrm{M}$ & $\mathrm{P}$ & 0 & $1 \mathrm{D}$ & 1 & - \\
\hline 13 & $51 / \mathrm{F}$ & $\mathrm{C}$ & $3 \mathrm{D}$ & $3 \mathrm{D}$ & 1 & - \\
\hline 14 & $24 / \mathrm{F}$ & $\mathrm{C}$ & $1 \mathrm{D}$ & $2 \mathrm{D}$ & 2 & - \\
\hline 15 & $32 / \mathrm{F}$ & $\mathrm{C}$ & $1 \mathrm{D}$ & $1 \mathrm{D}$ & 1 & + \\
\hline 16 & $27 / \mathrm{F}$ & $\mathrm{C}$ & $1 \mathrm{D}$ & $2 \mathrm{D}$ & 1 & + \\
\hline 17 & $13 / \mathrm{F}$ & $\mathrm{C}$ & $1 \mathrm{~F}$ & 0 & 1 & - \\
\hline 18 & $68 / \mathrm{F}$ & $\mathrm{C}$ & $2 \mathrm{D}$ & 0 & 1 & + \\
\hline 19 & $35 / \mathrm{M}$ & $\mathrm{C}$ & $2 \mathrm{~F}$ & 0 & 1 & + \\
\hline 20 & $18 / \mathrm{M}$ & $\mathrm{C}$ & 0 & $1 \mathrm{D}$ & 1 & - \\
\hline 21 & $16 / \mathrm{M}$ & $\mathrm{C}$ & $1 \mathrm{D}$ & $3 \mathrm{D}$ & 2 & + \\
\hline
\end{tabular}

M, male; F, female; C, low-grade central; P, parosteal; 1, weak; 2, moderate; 3 , strong; 0 , negative (less than $1 \%$ staining); $\mathrm{F}$, focal (>1-10\%); D, diffuse (>10-100\%); DD, dedifferentiation, +, present; - , absent.

The density of the color filling each box represents the intensity of immunostaining (see online for colour version).

markers, whereas the remaining six cases were labeled for CDK4 only. Low-grade central osteosarcomas were positive for MDM2 in $89 \%(8 / 9)$ of the cases, and for CDK4 in $67 \%$ (6/9) of the cases. Of lowgrade central osteosarcomas, $56 \%(5 / 9)$ were labeled for both markers. Three low-grade central osteosarcomas labeled for MDM2 only, and one tumor was positive for CDK4 only. Among eight tumors that showed dedifferentiation (whether synchronous or metachronous), six cases (75\%) expressed both MDM2 and CDK4. Among 15 tumors that showed no dedifferentiation, seven cases (47\%) expressed both MDM2 and CDK4. There was a trend of correlation between dedifferentiation and dual immunoexpression of MDM2 and CDK4, but it was not statistically significant (Fisher's exact test, $P=0.19$; StatMateIII; ATMS, Tokyo). In one case accessioned in 1970s (case 7), we found that staining of the first block yielded negative results for both markers, and the second block was processed to yield weak diffuse positive staining for CDK4. Table 3 summarizes the comparative immunohistochemical results of lowgrade osteosarcoma and benign lesions. In contrast to sarcomas, only 1 of the 40 benign lesions showed immunoreactivity for MDM2 or CDK4 (Figure 3 right and 4 right). The only immunopositive benign lesion was a bizarre parosteal osteochondromatous proliferation arising on the right ulna of a 41-year-old man, and it showed focal weak MDM2 staining and diffuse weak CDK4 staining. The immunopositive cells were mostly limited to osteoblastic cells rimming the lesional bone trabeculae, but intervening spindle cells were also labeled. A separate block of this case was prepared to confirm the immunoreactivity. Additional four cases (two myositides ossificans, one florid reactive periostitis, and one fibrous dysplasia) showed weak staining of either antibody in less than $1 \%$ of lesional cells in areas with myxoid/edematous change, and these signals were quantitatively regarded as negative in this study. The combination of MDM2 and CDK4 thus showed 100\% sensitivity and $97.5 \%$ specificity for the diagnosis of low-grade osteosarcoma.

\section{Influence of Decalcification on MDM2/CDK4 Immunohistochemistry}

Table 4 shows the intensity (weak, moderate, strong) and the extent (\%) of MDM2/CDK4 immunoreactivity in a dedifferentiated liposarcoma (a known positive control) after decalcification for a series of durations. CDK4 staining only mildly decreased in intensity in 1 week, and it retained diffuse staining pattern for 1 week; however, the extent of MDM2 staining was rapidly reduced in 1 day, but the labeled cells retained strong staining intensity for 2 weeks. A repeat experiment using a different case of dedifferentiated liposarcoma showed similar tendency of staining alterations in MDM2 and CDK4 (data not shown).

\section{Discussion}

In this analysis, we showed that all the studied low-grade osteosarcomas expressed MDM2 and/or CDK4 protein, whereas only one of the benign lesions labeled for these markers. The labeling of low-grade osteosarcoma is often diffuse that it would be readily recognizable even in a small biopsy specimen. The majority of the staining was moderate to strong in intensity, and it was readily seen at low magnification. Fifty-seven percent of low-grade osteosarcoma were labeled for both MDM2 and CDK4. Of note, MDM2 and CDK4 immunostaining was shown not only in the classic histological pattern of this sarcoma, but it was also observed in the known histological variety, including fibrous dysplasia-like, desmoplastic fibroma-like, and osteochondroma-like phenotypes, supporting the view that such morphological diver- 

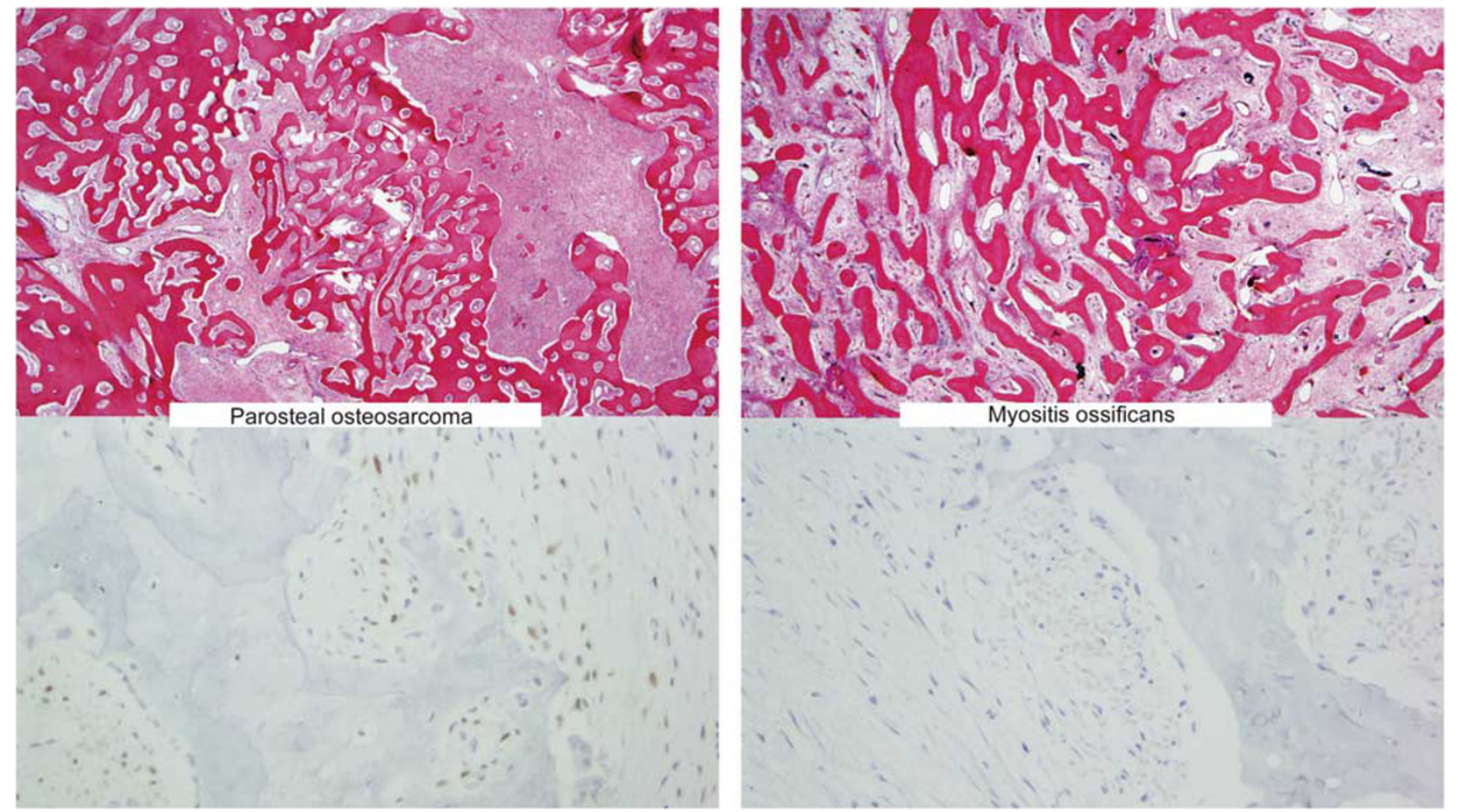

Figure 3 Comparative immunohistochemistry of a parosteal osteosarcoma (left: H\&E and moderate CDK4 immunostaining in case 2) and a myositis ossificans (right: H\&E and negative CDK4 immunostaining).
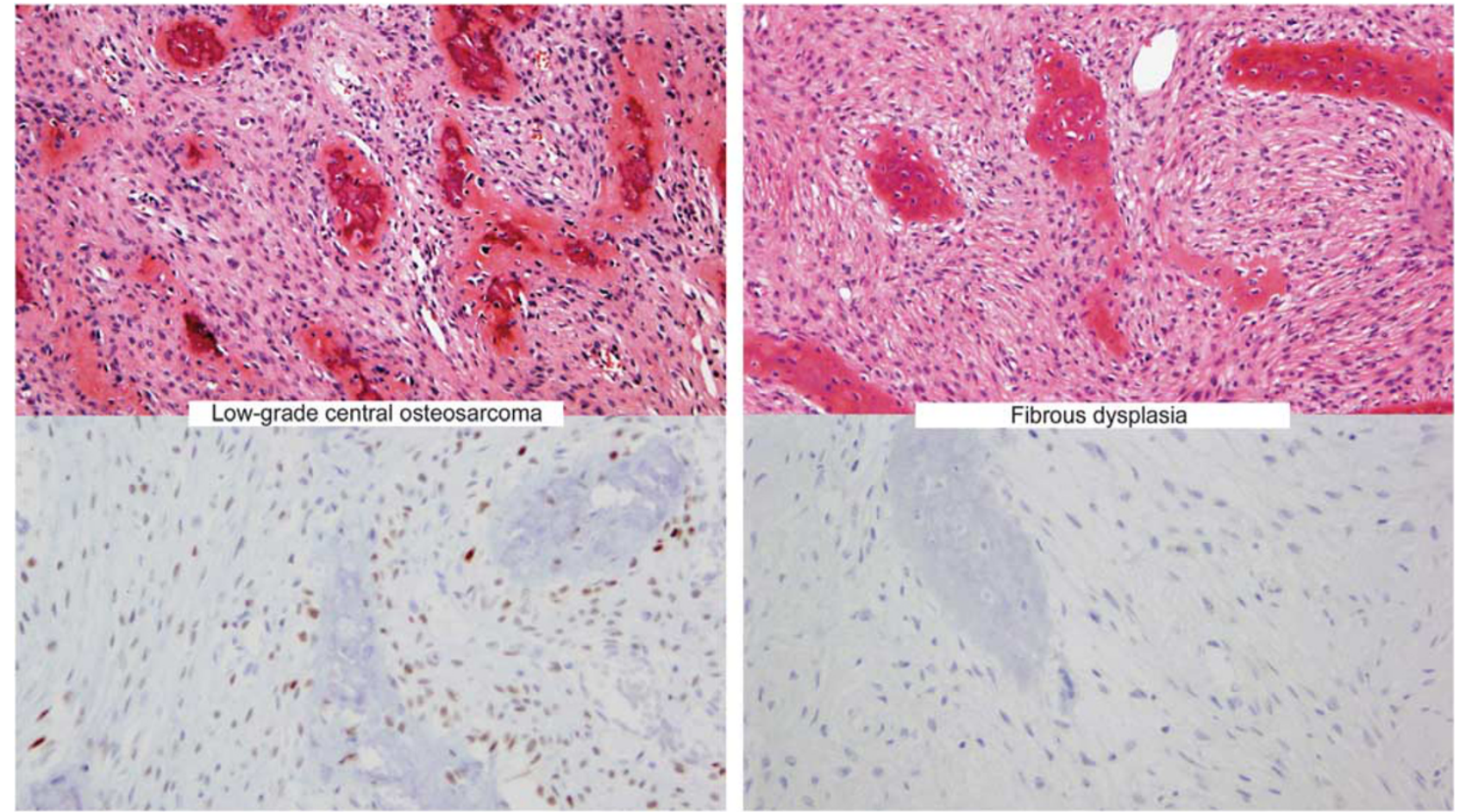

Figure 4 Comparative immunohistochemistry of a low-grade central osteosarcoma (left: H\&E and strong MDM2 immunostaining in case 13) and a fibrous dysplasia (right: H\&E and negative MDM2 immunostaining).

sity represents a spectrum of the same process. In contrast, the overwhelming majority of the benign lesions totally lack immunoreactivity for MDM2 or CDK4, with only a few cases showing staining of weak intensity (less than $1 \%$ of lesional cells in most cases). 


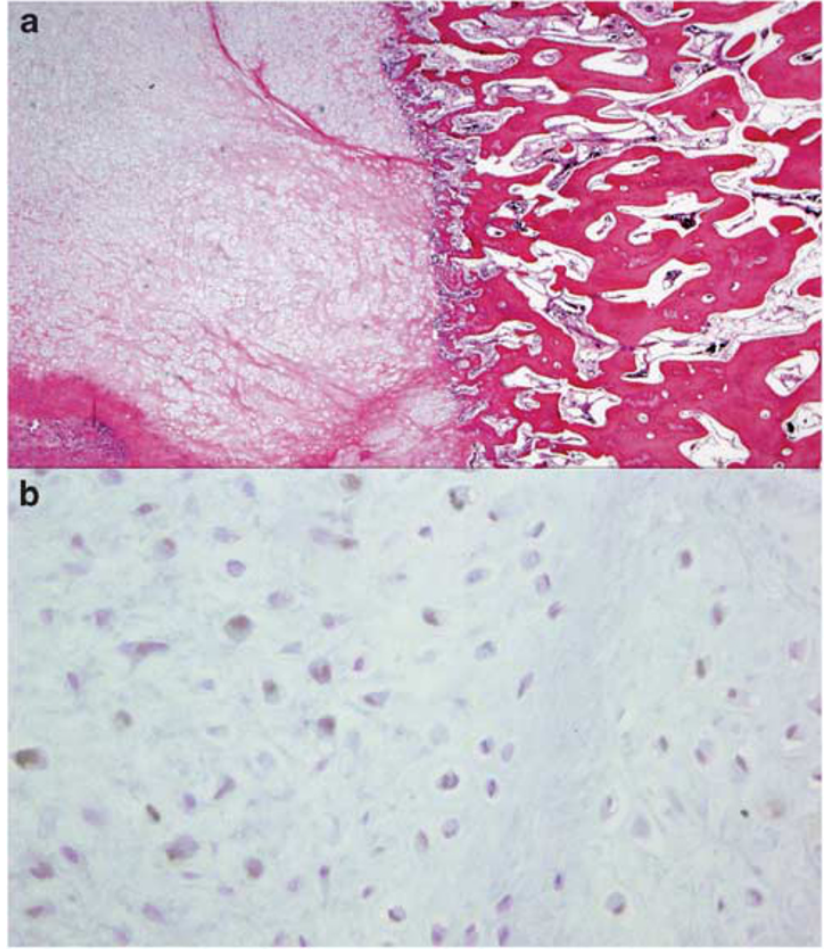

Figure 5 A parosteal osteosarcoma simulating osteochondroma shows weak CDK4 immunoreactivity in neoplastic chondrocytes (a: H\&E, b: CDK4 immunostaining, case 5b).

The MDM2 and CDK4 immunopositivity rate (70\% for MDM2 and $87 \%$ for CDK4) observed in this study was slightly higher than the previous reports. The explanation of the minor discordance includes technical differences such as antibody dilution and antigen retrieval method. In addition, our $1 \%$ cutoff for positivity is less strict than the previous studies, many of which required $10 \%$ staining to be designated as positive. This $1 \%$ cutoff was developed to maximize the distinguishing power of these immunostains between benign lesions and low-grade osteosarcomas. Staining of $1-10 \%$ of cells also regularly yielded an impression of focal, but significant, positivity (rather than negativity) to us, making this approach more intuitive than others. Yet another potential cause of discordant results among different studies may concern diagnostic criteria of low-grade osteosarcoma. The term 'low-grade' may have been applied in more than one way in different studies. For example, variants such as osteoblastoma-like osteosarcoma, ${ }^{5,32}$ chondromyxoid fibroma-like osteosarcoma, ${ }^{5}$ and sclerosing osteosarcoma with 'normalized' tumor cells ${ }^{5}$ may have been included in some studies as low-grade osteosarcoma, because of low nuclear grade and/or abundant matrix formation. In contrast, we have adhered to the view of the World Health Organization that variants such as osteoblastoma-like osteosarcoma should not be regarded as low-grade osteosarcoma; ${ }^{4}$ we have specifically limited the study subjects to fibroblastic

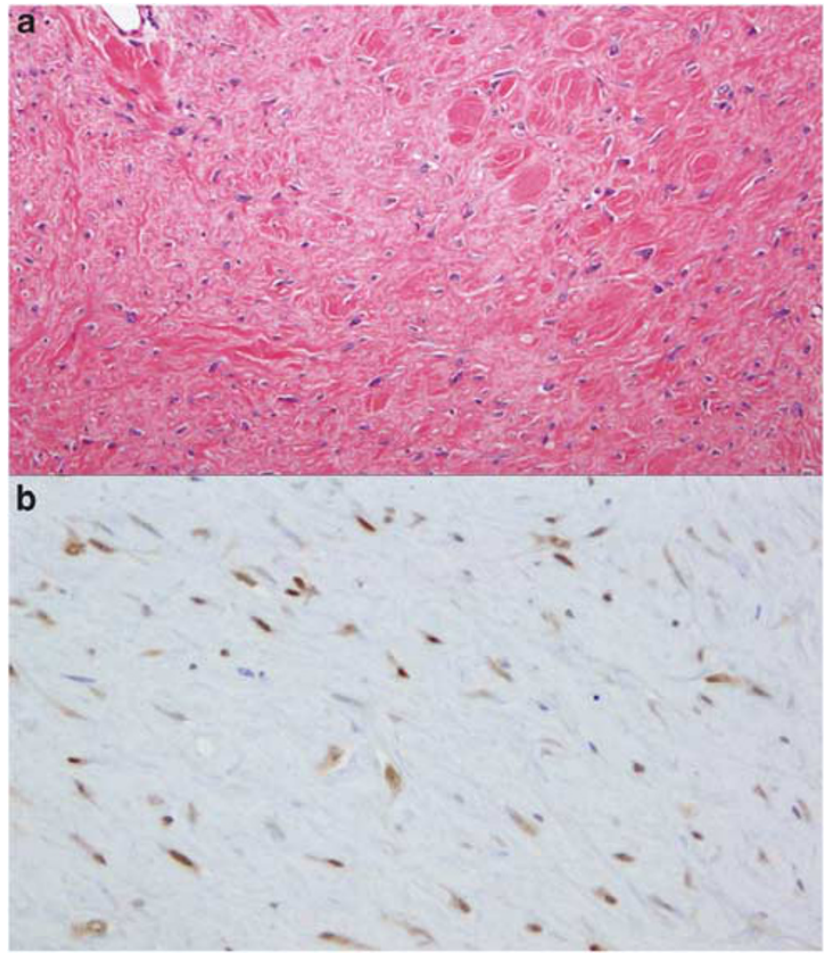

Figure 6 A parosteal osteosarcoma resembling desmoplastic fibroma shows strong CDK4 immunoreactivity (a: H\&E, b: CDK4 immunostaining, case 6).

Table 3 MDM2/CDK4 comparative immunohistochemistry

\begin{tabular}{lcc}
\hline Lesions & $\begin{array}{c}\text { Number of } \\
\text { immunopositive } \\
\text { cases /total } \\
\text { cases }\end{array}$ \\
\cline { 2 - 3 } & MDM2 & CDK4 \\
\hline Parosteal osteosarcoma & $8 / 14$ & $14 / 14$ \\
Low-grade central osteosarcoma & $8 / 9$ & $6 / 9$ \\
Myositis ossificans (heterotopic ossification) & $0 / 11$ & $0 / 11$ \\
Fibrous dysplasia & $0 / 14$ & $0 / 14$ \\
Osteochondroma & $0 / 6$ & $0 / 6$ \\
Florid reparative periostitis & $0 / 4$ & $0 / 4$ \\
Bizarre parosteal osteochondromatous & $1 / 3$ & $1 / 3$ \\
proliferation & & \\
Turret exostosis & $0 / 1$ & $0 / 1$ \\
Desmoplastic fibroma & $0 / 1$ & $0 / 1$ \\
& &
\end{tabular}

Table 4 Influence of decalcification on MDM2/CDK4 immunohistochemistry using a known positive control

\begin{tabular}{lllll}
\hline Antibody & \multicolumn{4}{c}{ Duration of decalcification } \\
\cline { 2 - 5 } & None & 1 day & 1 week & 2 weeks \\
& & & Strong, & Strong, \\
MDM2 & Strong, & Strong, & 20\% & $10 \%$ \\
& $90 \%$ & $20 \%$ & Moderate, & Weak, \\
CDK4 & Strong, & Strong, & Mod \\
& $95 \%$ & $95 \%$ & $90 \%$ & \\
\hline
\end{tabular}


low-grade osteosarcoma, and excluded osteoblastic or chondroblastic osteosarcomas from the study, regardless of nuclear grade or quantity of matrix.

Some of the low-grade osteosarcomas in our study dated back to 1960s. Although we noted that the cases accessioned before 1980s labeled more weakly than recent samples, old materials still showed diffuse immunoreactivity. This fact suggests that the immunostain in discussion may be applicable to remote archival materials and can be used when reviewing prior accessions to explain the perplexing recurrences of ostensibly benign lesions.

All the examined materials in the present analysis were decalcified. The decalcification methods were not standardized, and each specimen followed routine processing in different laboratories at different times. The solution used for most in-house specimens was Plank-Rychlo's; however, no information was available for consultation materials. We are aware that some resection specimens were heavily decalcified in toto for over 2 weeks. Our pilot study using two cases of dedifferentiated liposarcoma suggested that CDK4 staining is relatively resistant to decalcification for a short period of time, and that the extent of MDM2 staining can be significantly reduced by the process. It is notable that CDK4 showed a higher rate of positivity $(87 \%)$ compared with MDM2 (70\%) in low-grade osteosarcomas. In addition, among 10 cases for which only one marker was positive, CDK4 showed greater positivity (7 cases, $70 \%$ ). Although these results may indicate a more central role of CDK4 in tumorigenesis, alternatively, they may be attributable to the relative vulnerability of MDM2 antigenicity to short-term decalcification.

This study was inspired by the works performed in well-differentiated liposarcoma, a low-grade soft tissue sarcoma that frequently harbors MDM2 and CDK4 amplification, resulting in the overexpression of their protein products. Because the benign mimics of well-differentiated liposarcoma such as large lipomas lack amplification and overexpression of these oncogenes, the MDM2 and CDK4 immunostains were recommended as an ancillary tool to distinguish between well-differentiated liposarcoma and lipoma. ${ }^{33,34}$ This method has been thus far positively accepted by many soft tissue pathologists, and we, too, have found it helpful in the assessment of morphologically borderline cases of lipogenic tumors. Unlike well-differentiated liposarcoma, which co-expresses MDM2 and CDK4 in most of the examples, ${ }^{33}$ only $57 \%$ of the low-grade osteosarcomas expressed both of these markers in this analysis. In addition, although well-differentiated liposarcoma tends to show MDM2 more consistently than CDK $4,{ }^{33}$ we and others ${ }^{15,30}$ showed that lowgrade osteosarcomas were more commonly positive for CDK4 than MDM2. Such immunohistochemical differences between liposarcoma and osteosarcoma may reflect the actual difference in the prevalence of $M D M 2$ and $C D K 4$ gene amplification in each type of the tumors. Although we did not explore the genetic status in this study, it is suggested that the prevalence of amplification may be lower in lowgrade osteosarcoma ${ }^{15,19,35}$ than that reported in welldifferentiated liposarcoma. Alternatively, because MDM2 and CDK4 stainings appear to be influenced by decalcification, as implicated in our pilot study, MDM2 and CDK4 labeling pattern in this analysis may not have accurately reflected the genetic status in some materials. Further study is warranted to determine how complete the analogy is between the genetics and immunohistochemistry of well-differentiated liposarcoma and low-grade osteosarcoma.

In summary, we have shown that MDM2 and CDK4 were frequently immunoexpressed in lowgrade osteosarcoma, and that their expressions were virtually negative in the benign histological mimics of this sarcoma. The combination of these two markers may thus serve as a useful adjunct in the diagnosis of low-grade osteosarcoma. Further analysis on a larger series is required to see if this approach is reliably adoptable to routine diagnostic practice of bone tumors.

\section{Acknowledgements}

We thank Dr Tetsuo Imamura, Dr Tsuyoshi Ishida, and Dr Yuji Miura for providing pathological materials and/or clinical information. We also appreciate Ms Sachiko Miura and Ms Chizu Kina for superb technical assistance.

\section{Disclosure/conflict of interest}

The authors declare no conflict of interest.

\section{References}

1 Schwab JH, Antonescu CR, Athanasian EA, et al. A comparison of intramedullary and juxtacortical low-grade osteogenic sarcoma. Clin Orthop Relat Res 2008;466:1318-1322.

2 Dorfman HD, Czerniak B. Bone Tumors. Mosby: St Louis, 1998.

3 Unni KK, Inwards CY, Bridge JA, et al. Tumors of the Bones and Joints. ARP Press: Silver Spring, 2005.

4 Fletcher CDM, Unni KK, Mertens F, eds Pathology and Genetics of Tumours of Soft Tissue and Bone. IARC Press: Lyon, 2002.

5 Mirra JM, Picci P, Gold RH. Bone Tumors: Clinical, Radiologic, and Pathologic Correlations. Lea \& Febiger: Philadelphia, 1989.

6 Okada K, Frassica FJ, Sim FH, et al. Parosteal osteosarcoma. A clinicopathological study. J Bone Joint Surg Am 1994;76:366-378.

7 Kurt AM, Unni KK, McLeod RA, et al. Low-grade intraosseous osteosarcoma. Cancer 1990;65:1418-1428.

8 Sheth DS, Yasko AW, Raymond AK, et al. Conventional and dedifferentiated parosteal osteosarcoma. Diagnosis, treatment, and outcome. Cancer 1996;78:2136-2145. 
9 Orndal C, Mandahl N, Rydholm A, et al. Supernumerary ring chromosomes in five bone and soft tissue tumors of low or borderline malignancy. Cancer Genet Cytogenet 1992;60:170-175.

10 Sinovic JF, Bridge JA, Neff JR. Ring chromosome in parosteal osteosarcoma. Clinical and diagnostic significance. Cancer Genet Cytogenet 1992;62:50-52.

11 Gisselsson D, Palsson E, Hoglund M, et al. Differentially amplified chromosome 12 sequences in low- and high-grade osteosarcoma. Genes Chromosomes Cancer 2002;33:133-140.

12 Szymanska J, Mandahl N, Mertens F, et al. Ring chromosomes in parosteal osteosarcoma contain sequences from 12q13-15: a combined cytogenetic and comparative genomic hybridization study. Genes Chromosomes Cancer 1996;16:31-34.

13 Tarkkanen M, Karhu R, Kallioniemi A, et al. Gains and losses of DNA sequences in osteosarcomas by comparative genomic hybridization. Cancer Res 1995; 55:1334-1338.

14 Tarkkanen M, Bohling T, Gamberi G, et al. Comparative genomic hybridization of low-grade central osteosarcoma. Mod Pathol 1998;11:421-426.

15 Gamberi G, Ragazzini P, Benassi MS, et al. Analysis of 12q13-15 genes in parosteal osteosarcoma. Clin Orthop Relat Res 2000;377:195-204.

16 Wunder JS, Eppert K, Burrow SR, et al. Co-amplification and overexpression of CDK4, SAS and MDM2 occurs frequently in human parosteal osteosarcomas. Oncogene 1999;18:783-788.

17 Noble-Topham SE, Burrow SR, Eppert K, et al. SAS is amplified predominantly in surface osteosarcoma. J Orthop Res 1996;14:700-705.

18 Wei G, Lonardo F, Ueda T, et al. CDK4 gene amplification in osteosarcoma: reciprocal relationship with INK4A gene alterations and mapping of 12q13 amplicons. Int J Cancer 1999;80:199-204.

19 Park HR, Jung WW, Bertoni F, et al. Molecular analysis of p53, MDM2 and H-ras genes in low-grade central osteosarcoma. Pathol Res Pract 2004;200: 439-445.

20 Lopez-Guerrero JA, Lopez-Gines C, Pellin A, et al. Deregulation of the $G_{1}$ to $S$-phase cell cycle checkpoint is involved in the pathogenesis of human osteosarcoma. Diagn Mol Pathol 2004;13:81-91

21 Bertoni F, Bacchini P, Fabbri N, et al. Osteosarcoma. Low-grade intraosseous-type osteosarcoma, histologically resembling parosteal osteosarcoma, fibrous dysplasia, and desmoplastic fibroma. Cancer 1993;71: $338-345$.
22 Lin J, Yao L, Mirra JM, et al. Osteochondroma like parosteal osteosarcoma: a report of six cases of a new entity. AJR Am J Roentgenol 1998;170:1571-1577.

23 Dorfman HD, Ishida T, Tsuneyoshi M. Exophytic variant of fibrous dysplasia (fibrous dysplasia protuberans). Hum Pathol 1994;25:1234-1237.

24 Meneses MF, Unni KK, Swee RG. Bizarre parosteal osteochondromatous proliferation of bone (Nora's lesion). Am J Surg Pathol 1993;17:691-697.

25 de Silva MV, Reid R. Myositis ossificans and fibroosseous pseudotumor of digits: a clinicopathological review of 64 cases with emphasis on diagnostic pitfalls. Int J Surg Pathol 2003;11:187-195.

26 Bahk WJ, Mirra JM. Pseudoanaplastic tumors of bone. Skeletal Radiol 2004;33:641-648.

27 Okada K, Nishida J, Morita T, et al. Low-grade intraosseous osteosarcoma in northern Japan: advantage of AgNOR and MIB-1 staining in differential diagnosis. Hum Pathol 2000;31:633-639.

28 Pollandt K, Engels C, Kaiser E, et al. Gsalpha gene mutations in monostotic fibrous dysplasia of bone and fibrous dysplasia-like low-grade central osteosarcoma. Virchows Arch 2001;439:170-175.

29 Lopes MA, Nikitakis NG, Ord RA, et al. Amplification and protein expression of chromosome 12q13-15 genes in osteosarcomas of the jaws. Oral Oncol 2001;37:566-571.

30 Ragazzini P, Gamberi G, Benassi MS, et al. Analysis of $S A S$ gene and CDK4 and MDM2 proteins in low-grade osteosarcoma. Cancer Detect Prev 1999;23:129-136.

31 Unni KK, Dahlin DC. Grading of bone tumors. Semin Diagn Pathol 1984;1:165-172.

32 Dorfman HD, Weiss SW. Borderline osteoblastic tumors: problems in the differential diagnosis of aggressive osteoblastoma and low-grade osteosarcoma. Semin Diagn Pathol 1984;1:215-234

33 Binh MB, Sastre-Garau X, Guillou L, et al. MDM2 and CDK4 immunostainings are useful adjuncts in diagnosing well-differentiated and dedifferentiated liposarcoma subtypes: a comparative analysis of 559 soft tissue neoplasms with genetic data. Am J Surg Pathol 2005;29:1340-1347.

34 Pilotti S, Della Torre G, Mezzelani A, et al. The expression of MDM2/CDK4 gene product in the differential diagnosis of well differentiated liposarcoma and large deep-seated lipoma. Br J Cancer 2000;82:1271-1275.

35 Mejia-Guerrero S, Quejada M, Gokgoz N, et al. Characterization of the $12 q 15$ MDM2 and 12q13-14 CDK4 amplicons and clinical correlations in osteosarcoma. Genes Chromosomes Cancer 2010;49:518-525. 